\title{
ON RHOADES' OPEN QUESTIONS AND SOME FIXED POINT THEOREMS FOR A CLASS OF MAPPINGS
}

\author{
SHIH - SEN CHANG ${ }^{1}$
}

\begin{abstract}
In this paper the concept of $C$-mapping and some fixed point theorems for such mappings are introduced and presented.

These results are then used to answer two questions of Rhoades.
\end{abstract}

1. Introduction. Let $(X, d)$ be a complete metric space and $T$ a mapping from $X$ into $X . T$ is called a Rhoades mapping if for any $x, y \in X, x \neq y$,

$$
d(T x, T y)<\max \{d(x, y), d(x, T x), d(y, T y), d(x, T y), d(y, T x)\} .
$$

Concerning the existence of a fixed point for such mappings $T$, Rhoades [4] pointed out that the condition

$\mathrm{A}_{1} . T$ is continuous and $\left\{T^{n} x_{0}\right\}_{n=0}^{\infty}$ has a cluster point for some $x_{0} \in X$, is needed in order to ensure that every such $T$ possesses a fixed point.

The following open questions were raised by Rhoades [4] (also see [5]).

(1) If $T$ is a Rhoades mapping satisfying condition $\mathrm{A}_{1}$, does $T$ possess a fixed point?

(2) If the answer to (1) is no, then what additional hypotheses on $T$ or $X$ are needed to guarantee the existence of a fixed point?

Recently, some answers to these questions were obtained. In [6] it was shown that the answer to (1) is no. Using a different example, (1) was also answered in the negative in [7]. For (2) a partial answer is that the condition

$\mathrm{A}_{2} . T$ is continuous and $X$ is compact, is sufficient. (See [8, Theorem 1].)

The purpose of this paper is to introduce the concept of a $C$-mapping and to establish necessary and sufficient conditions for such mappings to possess a fixed point. Using these results Rhoades' open questions are answered.

2. Definition and preliminaries. Throughout this paper we always assume that $(X, d)$ is a complete metric space, $T$ a self-map of $X . T$ is called a $C$-mapping if for any $x \in X$ and any positive integer $n \geqslant 2$ satisfying

$$
T^{i} x \neq T^{j} x, \quad 0 \leqslant i<j \leqslant n-1,
$$

Received by the editors December 21, 1984 and, in revised form, April 2, 1985.

1980 Mathematics Subject Classification. Primary 54H25.

Key words and phrases. C-mapping, Rhoades' open question, fixed point theorems.

${ }^{1}$ Research supported by the Science Fund of the Chinese Academy of Sciences. 
we have

$$
d\left(T^{n} x, T^{i} x\right)<\max _{1 \leqslant j \leqslant n}\left\{d\left(T^{j} x, x\right)\right\}, \quad i=1,2, \ldots, n-1 .
$$

REMARK 1. $C$-mappings are a class of important mappings containing a number of well-known mappings as special cases. For example, the mappings numbered (1)-(25) by Rhoades [4] are all special cases of $C$-mappings. In particular, the mappings listed below are special cases of a $C$-mapping.

(1) Banach's contraction mapping $T$ [1]: i.e., there exists a number $h \in(0,1)$ such that

$$
d(T x, T y) \leqslant h d(x, y), \quad \forall x, y \in X .
$$

(2) Kannan's mapping $T$ [3]: i.e., there exists a number $h \in\left(0, \frac{1}{2}\right)$ such that

$$
d(T x, T y) \leqslant h\{d(x, T x)+d(y, T y)\}, \quad \forall x, y \in X .
$$

(3) Ciric's mapping $T$ [2]: i.e., there exists a constant $h \in(0,1)$ such that for any $x, y \in X$,

(2.5) $d(T x, T y) \leqslant h \max \{d(x, y), d(x, T x), d(y, T y), d(x, T y), d(y, T x)\}$.

(4) Rhoades' mapping $T$ [4].

As is well known, mappings (1), (2), and (3) are all special cases of mapping (4). Therefore, in order to prove this statement it suffices to prove that the Rhoades mapping $T$ is a special case of a $C$-mapping.

Let $x \in X$ and $n \geqslant 2$ be such that (2.1) is satisfied. If $T$ is a Rhoades mapping, it can be shown by induction that (2.2) is true. In fact, for $n=2$, from (1.1),

$$
\begin{aligned}
d\left(T^{2} x, T x\right) & <\max \left\{d(T x, x), d\left(T x, T^{2} x\right), d(x, T x), d(T x, T x), d\left(x, T^{2} x\right)\right\} \\
& =\max \left\{d(T x, x), d\left(T^{2} x, x\right)\right\}
\end{aligned}
$$

and (2.2) is satisfied.

Suppose that (2.2) is true for $n=k-1, k \geqslant 3$, and denote

$$
\alpha=\max _{1 \leqslant j \leqslant k-1}\left\{d\left(T^{j} x, x\right)\right\} .
$$

By the induction hypothesis and condition (1.1),

$$
\begin{aligned}
& d\left(T^{k} x, T^{k-1} x\right)<\max \{ d\left(T^{k-1} x, T^{k-2} x\right), d\left(T^{k-1} x, T^{k} x\right), \\
&\left.d\left(T^{k-2} x, T^{k-1} x\right), d\left(T^{k-1} x, T^{k-1} x\right), d\left(T^{k-2} x, T^{k} x\right)\right\} \\
& \leqslant \max \left\{\alpha, d\left(T^{k} x, T^{k-2} x\right)\right\} .
\end{aligned}
$$

Also by induction it can be shown that

$$
d\left(T^{k} x, T^{k-i} x\right)<\max \left\{\alpha, d\left(T^{k} x, T^{k-i-1} x\right)\right\}, \quad i=1,2, \ldots, k-1 .
$$

Therefore

$$
d\left(T^{k} x, T x\right)<\max \left\{\alpha, d\left(T^{k} x, x\right)\right\}=\max _{1 \leqslant j \leqslant k}\left\{d\left(T^{j} x, x\right)\right\}
$$

and successively,

$$
d\left(T^{k} x, T^{i} x\right)<\max _{1 \leqslant j \leqslant k}\left\{d\left(T^{j} x, x\right)\right\}, \quad i=1,2, \ldots, k-1 .
$$




\section{Main results.}

Theorem 1. Let $T$ be a $C$-mapping on $X$. Then $T$ has a fixed point in $X$ if and only if there exist integers $m$ and $n, m>n \geqslant 0$, and a point $x \in X$ such that

$$
T^{m} x=T^{n} x
$$

If this condition is satisfied, then $T^{n} x$ is a fixed point of $T$.

Proof. Necessity. Let $x^{*} \in X$ be a fixed point of $T$; i.e., $x^{*}=T x^{*}$. Then (3.1) is true with $m=1$ and $n=0$.

Sufficiency. Suppose there exists a point $x \in X$ and two integers $m, n, m>n \geqslant 0$, such that $T^{m} x=T^{n} x$. Without loss of generality, we can assume that $m$ is the minimal such integer satisfying $T^{k} x=T^{n} x, k>n$. Putting $y=T^{n} x$ and $p=m-$ $n$, we have $T^{p} y=y$, and $p$ is the minimal integer satisfying $T^{k} y=y, k \geqslant 1$.

To show that $y$ is a fixed point of $T$, assume that it is not. Then $p \geqslant 2$, and

$$
T^{i} y \neq T^{j} y \quad \text { for } 0 \leqslant i<j \leqslant p-1 .
$$

Since $T$ is a $C$-mapping,

$$
\begin{aligned}
d\left(y, T^{i} y\right) & =d\left(T^{p} y, T^{i} y\right)<\max _{1 \leqslant j \leqslant p}\left\{d\left(T^{j} y, y\right)\right\} \\
& =\max _{1 \leqslant j \leqslant p-1}\left\{d\left(T^{j} y, y\right)\right\}, \quad i=1,2, \ldots, p-1 .
\end{aligned}
$$

This implies that

$$
\max _{1 \leqslant j \leqslant p-1}\left\{d\left(y, T^{i} y\right)\right\}<\max _{1 \leqslant j \leqslant p-1}\left\{d\left(T^{j} y, y\right)\right\},
$$

which is a contradiction. Therefore $y=T^{n} x$ is a fixed point of $T$.

Lemma. Let $T$ be a continuous $C$-mapping on $X$. Let $x$ be a point in $X$ for which $\left\{T^{n} x\right\}_{n=0}^{\infty}$ has a cluster point $x_{0}$. Then $T^{n} x_{0}, n=0,1,2, \ldots$ are also cluster points of $\left\{T^{n} x\right\}_{n=0}^{\infty}$.

Proof. Since $x_{0}$ is a cluster point of $\left\{T^{n} x\right\}_{n=0}^{\infty}$, there exists a subsequence $\left\{T^{n_{i}} x\right\} \subset\left\{T^{n} x\right\}$ which converges to $x_{0}$. Since $T$ is continuous,

$$
\lim _{i \rightarrow \infty} T^{n_{i}+k} x=T^{k} x_{0}, \quad k=0,1,2, \ldots
$$

Theorem 2. Let $T$ be a continuous $C$-mapping on $X$. Let $x$ be a point in $X$ for which $\left\{T^{n} x\right\}_{n=0}^{\infty}$ has a cluster point $x_{0}$. Then $T$ has a fixed point in $\left\{T^{n} x_{0}\right\}_{n=0}^{\infty}$ if and only if one of the following conditions is satisfied:

(i) $\left\{T^{n} x\right\}_{n=0}^{\infty}$ is convergent.

(ii) There exists a positive integer $m$ such that $T^{m} y=y$, where $y$ is some point in $\left\{T^{n} x_{0}\right\}_{n=0}^{\infty}$.

Proof. Necessity. If $\left\{T^{n} x_{0}\right\}=\left\{x_{0}\right\}$, then it is obvious that $\left\{T^{n} x\right\}$ is convergent, and (i) is satisfied. If $\left\{T^{n} x_{0}\right\}$ is not the singleton $x_{0}$, let $y \in\left\{T^{n} x_{0}\right\}$ be a fixed point of $T$. Since $y$ is a cluster point of $\left\{T^{n} x\right\}$, there exists a subsequence $\left\{T^{n_{i}} x\right\}$ converging to $y$. Thus

$$
\lim _{i \rightarrow \infty} T^{n_{i}+k} x=T^{k} y=y, \quad k=0,1,2, \ldots,
$$

and (ii) is satisfied. 
Sufficiency. If (i) is satisfied, then $\left\{T^{n} x_{0}\right\}$ is the singleton $x_{0}$ and $x_{0}$ is a fixed point of $T$. If (ii) is satisfied, then $T$ has a fixed point by Theorem 1 .

The following results are immediate consequences of Theorems 1 and 2 .

Corollary 1. Let $T$ be a Rhoades mapping on $X$. Then $T$ has a fixed point in $X$ if and only if there exist integers $m$ and $n, m>n \geqslant 0$ and a point $x \in X$ such that $T^{m} x=T^{n} x$. (If this condition is satisfied then $T^{n} x$ is the unique fixed point of $T$.)

Corollary 2. Let $T$ be a continuous Rhoades mapping on $X$. Let $\left\{T^{n} x\right\}_{n=0}^{\infty}$ have a cluster point $x_{0}$ for some $x \in X$. Then $T$ has a fixed point in $\left\{T^{n} x_{0}\right\}_{n=0}^{\infty}$ if and only if one of the following conditions holds:

(i) $\left\{T^{n} x\right\}$ is convergent.

(ii) There exists a positive integer $m$ such that $T^{m} y=y$, where $y$ is some point of $\left\{T^{n} x_{0}\right\}_{n=0}^{\infty}$.

REMARK 2. Corollary 2 gives an immediate answer to Rhoades' questions.

ACKNOWLEDGEMENT. I would like to express my thanks to the referee for his helpful suggestions.

\section{REFERENCES}

1. S. Banach, Théorie des opérations linéaires, PWN, Warszawa, 1932.

2. L. B. Ciric, A generalization of Banach's contraction principle, Proc. Amer. Math. Soc. 45 (1974), 267-273.

3. R. Kannan, Some results on fixed points. II, Amer. Math. Monthly 76 (1969), 405-408.

4. B. E. Rhoades, $A$ comparison of various definitions of contractive mappings, Trans. Amer. Math. Soc. 226 (1977), 257-290.

5. Contractive definitions revisited, Topological Methods in Nonlinear Functional Analysis, Contemporary Math., Vol. 21, Amer. Math. Soc., Providence, R. I., 1983, pp. 189-205.

6. J. Kincses and V. Totik, Theorems and counterexamples on contractive mappings, preprint.

7. L. E. Taylor, A contractive mapping without fixed points, Notices Amer. Math. Soc. 24 (1977), A-649.

8. Sehie Park, On general contractive type conditions, J. Korean Math. Soc. 17 (1980), 131-140.

Department of Mathematics, Sichuan University, Chengdu, Sichuan 610064, People's RepubLIC OF CHINA 